\title{
Predicting Virologic Failure Among HIV-1-Infected Children Receiving Antiretroviral Therapy in Tanzania: a Cross-Sectional Study
}

\author{
Susan D. Emmett, BA, * + t Coleen K. Cunningham, MD, $\S$ Blandina T. Mmbaga, MD, Mmed, \\ Grace D. Kinabo, MD, Mmed," Werner Schimana, MD, $\mid$ Mark E. Swai, MD, Mmed,** \\ John A. Bartlett, MD, †\# John A. Crump, MB, ChB, DTM\&H, †\#**†† and Elizabeth A. Reddy, MD\#**
}

\begin{abstract}
Background: Many HIV care and treatment programs in resourcelimited settings rely on clinical and immunologic monitoring of antiretroviral therapy (ART), but accuracy of this strategy to detect virologic failure (VF) among children has not been evaluated.

Methods: A cross-sectional sample of HIV-infected children aged $1-16$ years on ART $\geq 6$ months receiving care at a Tanzanian referral center underwent clinical staging, CD4 lymphocyte measurement, plasma HIV-1 RNA level, and complete blood count. Associations with VF (HIV-1 RNA $\geq 400$ copies $/ \mathrm{mL}$ ) were determined utilizing bivariable and multivariate analyses; accuracy of current clinical and immunologic guidelines in identifying children with VF was assessed.
\end{abstract}

Received for publication September 16, 2009; accepted December 11, 2009. From the *Duke University Medical Center, Durham, North Carolina, USA; $\dagger$ Duke Global Health Institute, Duke University, Durham, North Carolina, USA; $\$$ Howard Hughes Medical Institute Research Training Fellowships for Medical Students, Bethesda, Maryland, USA; §Department of Pediatrics, Division of Infectious Diseases, Duke University Medical Center, Durham, North Carolina, USA; "Department of Pediatrics, Kilimanjaro Christian Medical Centre, Moshi, Tanzania; \Elizabeth Glaser Pediatric AIDS Foundation, Moshi, Tanzania; \#Department of Internal Medicine, Division of Infectious Diseases, Duke University Medical Center, Durham, North Carolina, USA; **Kilimanjaro Christian Medical Centre, Moshi, Tanzania; and $\dagger \dagger$ Kilimanjaro Christian Medical College, Tumaini University, Moshi, Tanzania.

The study was supported by the Duke Center for AIDS Research an NIH funded program (P30 AI 64518). Additional investigator support was obtained from the AIDS Research and Training Grant (T32 AI007392-18, Dr. Reddy), Fogarty International Center (D43 PA-03-018, Dr. Crump), the Duke Clinical Trials Unit and Clinical Research Sites (U01 AI0698401, Dr. Crump), and the Duke University Center for AIDS Research, and International Studies on AIDS Associated Coinfections award (ISAAC) (U01 AI-03-036, Dr. Crump). Ms. Emmett was funded by a Howard Hughes Medical Institute Research Training Fellowship.

Presented at the First International Conference on Pediatric Virology Education [abstract TuPe7.1C09], 2009, Cape Town, South Africa.

Drs. J.A.B. and W.S. have received speaking honoraria from Abbott laboratories, and Dr. J.A.B. served as a consultant to Abbott laboratories, Merck, and Argos. All other authors declare no conflicts of interest with regards to this article.

Correspondence to: Elizabeth A. Reddy, MD, Child Centered Family Care Clinic, Duke University - Kilimanjaro Christian Medical Centre Collaboration, BOX 3010, Moshi, Tanzania (e-mail: reddy009@mc.duke.edu).

Copyright (C) 2010 by Lippincott Williams \& Wilkins
Findings: Of 206 children (median age 8.7 years, ART duration 2.4 years), $65(31.6 \%)$ demonstrated VF at enrollment. Clinical and immunological criteria identified 2 (3.5\%) of 57 children with VF on first-line therapy, exhibiting 3.5\% sensitivity and $100 \%$ specificity. VF was associated with younger age, receipt of nevirapine vs. efavirenz-based regimen, CD4\% $<25 \%$, and physician documentation of maladherence ( $P<0.05$ on bivariable analysis); the latter 2 factors remained significant on multivariate logistic regression.

Interpretation: This study demonstrates poor performance of clinical and immunologic criteria in identifying children with virologic failure. Affordable techniques for measuring HIV-1 RNA level applicable in resource-limited settings are urgently needed.

Key Words: Africa, antiretroviral therapy, HIV, pediatrics, virologic failure

(J Acquir Immune Defic Syndr 2010;54:368-375)

\section{INTRODUCTION}

Access to antiretroviral therapy (ART) has rapidly expanded in resource-limited regions since 2004. In Tanzania, use of ART has increased from less than $1 \%$ of those meeting eligibility criteria in 2004 to $31 \%$ in 2007, including over 11,000 children. ${ }^{1}$ With expanded access, programs in resource-limited settings are measuring reductions in morbidity and mortality similar to that achieved in highincome countries. ${ }^{2-5}$

However, substantial challenges for care and treatment programs remain; primary among them is limited availability of monitoring tools for measuring response to ART. ${ }^{6,7}$ Plasma HIV-1 RNA level is central to ART management in highincome countries, but cost and technical challenges make such monitoring unavailable to the majority of patients in resourcepoor settings such as Tanzania.,8 The World Health Organization (WHO) advocates a public health approach to ART, recognizing the potential role for plasma HIV-1 RNA testing but recommending clinical and immunological monitoring in most situations. ${ }^{9,10}$ However, little work has been done to assess the sensitivity and specificity of clinical and immunologic criteria to predict virologic failure, and 
we are aware of no other study evaluating these criteria in children. ${ }^{11-16}$

Effective monitoring methods may be particularly important to the long-term success of ART in resource-limited settings as there are limited options for second-line therapy and an increased risk of resistance mutations and subsequent second-line failure in patients who remain on failing regimens. ${ }^{17-19}$ Thus, methods for accurately detecting virologic failure are urgently needed. This study evaluates the effectiveness of clinical and immunologic criteria to ascertain virologic failure in children and supplements currently recommended criteria in situations where plasma HIV-1 RNA testing is not available.

\section{MATERIALS AND METHODS}

\section{Study Design and Participants}

This cross-sectional cohort study with retrospective chart review enrolled HIV-infected children receiving medical care at the Child Centered Family Care Clinic (CCFCC) of the Kilimanjaro Christian Medical Centre (KCMC), a referral hospital in northern Tanzania. KCMC distributes ART through funding from the President's Emergency Fund for AIDS Relief, and was the first site in northern Tanzania to offer free ART to children, starting in January, 2005. Patients initially paid 1000 Tanzanian shillings (equivalent to approximately 0.78 USD by November 2009 exchange rates) at each clinic visit; this fee was waived in 2007 and pediatric HIV care has since been provided free of charge.

ART at the CCFCC is provided according to standards defined by the Tanzanian National Guidelines for the Management of HIV and AIDS. ${ }^{20}$ First-line regimens are non-nucleoside reverse transcriptase inhibitor-based and consist of a combination of either nevirapine or efavirenz with zidovudine and lamivudine or stavudine and lamivudine. Second-line regimens are prescribed for children who meet immunologic and/or clinical criteria for regimen failure according to the Tanzanian National Guidelines. Second-line therapy consists of lopinavir/ritonavir with either abacavir and didanosine or zidovudine and lamivudine. Regimen changes between combinations of first-line medications, or changes to a protease inhibitor regimen due to toxicity or side effects from nevirapine and efavirenz, were still considered first line for the purposes of this analysis. The study was observational and any medication changes were at the discretion of clinicians.

Study inclusion criteria were aged 12 months to 16 years and treatment with ART for at least 6 months. Children less than 18 months at study enrollment had documentation of an HIV-1 RNA level $>5000$ copies per milliliter, CD4\% $<25 \%$, or a WHO Stage III or IV clinical event before ART initiation.

Informed consent was obtained from the parent or guardian for all participants and assent from the child if over 13 years of age. A standardized questionnaire was administered in Kiswahili by a social worker and by a physician to determine sociodemographic information and past medical history, including exposure to antiretroviral medications as part of prevention of maternal to child transmission programs, date of ART initiation, current ART regimen, medication changes, and tuberculosis treatment. Clinical staging was based on physician documentation of conditions included in the 2006 WHO Staging Criteria. ${ }^{21}$ All post-ART CD4 lymphocyte measurements and complete blood count (CBC) results, pre-ART nadir CD4 lymphocyte measurement, and monthly height and weight measurements were recorded through chart review. Children who met combined clinical and immunologic recommendations for regimen change according to WHO guidelines (Table 1) were designated as failing therapy by clinical and immunologic criteria., 22

Missed doses and treatment interruptions were assessed by physician documentation on the national standardized HIV care flow sheet - which is filled by clinicians monthly during follow up visits - and by asking the caregiver during the study enrollment visit to recall the last time a dose of medication was ever missed, how many doses were missed in the last month, and whether treatment had ever been stopped for a full day or more. Maladherence by physician history was defined as documentation on the HIV care flow sheet of at least 2 separate visits in which a family reported that the child missed more than 1 dose in the previous month, including any skipped or late clinic appointments resulting in delayed refill of medications. Family report of maladherence was defined as caregiver recall of more than 1 dose missed in the past month or any treatment interruption of at least 1 day. Disclosure of HIV status was assessed by questioning caregivers privately as to whether they had informed the child that she or he had HIV infection, that she or he had an illness (eg, of the blood or immune system) without ever specifying HIV, or that they had

TABLE 1. Summary of WHO Recommendations for Clinical and Immunological Monitoring of ART in Children in Areas Where CD4 Lymphocyte Count Is Available 9,22

\begin{tabular}{|c|c|}
\hline Clinical status at least 6 months post ART initiation & CD4 measurement(s) at least 6 months post ART initiation \\
\hline $\begin{array}{l}\text { Clinically well or new, relapsed or worsening WHO } \\
\text { Stage I or II event(s) }\end{array}$ & $\begin{array}{l}\text { Consider regimen change if two or more CD4 counts below age-related } \\
\text { threshold for severe immunodeficiency, }{ }^{*} \text { but regimen change particularly } \\
\text { recommended if CD4 declines further } \dagger\end{array}$ \\
\hline New, relapsed, or worsening WHO Stage III event(s) & $\begin{array}{l}\text { Recommend regimen change if CD4 count below age-related threshold } \\
\text { for severe immunodeficiency }\end{array}$ \\
\hline New, relapsed, or worsening WHO Stage IV event(s) & $\begin{array}{l}\text { One CD4 count below age-related threshold for severe immune deficiency } \\
\text { is recommended but not necessary to advise regimen change }\end{array}$ \\
\hline
\end{tabular}

*Age-related threshold for severe immune deficiency CD4\% $<20 \%$ or CD4 $<750$ cells per milliliter ( $12-35$ months), CD4\% $<20 \%$ or CD4 $<350$ cells per milliliter ( $36-59$ months), CD4\% $<15 \%$ or CD4 $<200$ cells per milliliter ( $\geq 5$ years).

$\dagger$ Regimen change particularly recommended if CD4\% $<15 \%$ (12-35 months of age), CD4\% $<10 \%$ (36-59 months), and CD4 $<100$ cells per milliliter ( $\geq 5$ years). 
never specifically discussed the child's illness. Analyses of disclosure were restricted to children aged 7 or older.

\section{Laboratory Assessments}

Five milliliters of whole blood were collected from each patient on the day of enrollment for CBC (Abbott Cell Dyn 3500 Hematology Analyzer, Abbott Diagnostics, Des Plaines, IL), CD4 lymphocyte measurement (FACSCalibur flow cytometry, Beckman, Dickinson, and Co., Franklin Lakes, NJ), and plasma HIV-1 RNA level (Abbott m2000 system, Abbott Molecular, Des Plaines, IL). ${ }^{23}$ The lower limit of detection for plasma HIV-1 RNA PCR was 40 copies per milliliter for $600 \mathrm{uL}$ samples and 150 copies per milliliter for $200 \mathrm{uL}$ sample volumes.

Enrollment laboratory testing was performed at the KCMC Biotechnology Laboratory, which participates in External Quality Assurance programs including United Kingdom National External Quality Assurance Scheme, College of American Pathologists, and the AIDS Clinical Trials Group Viral Quality Assurance Program. Prior CD4 lymphocyte measurements and $\mathrm{CBC}$ results abstracted from charts were performed at a variety of clinical laboratories, including KCMC Clinical Laboratory and other district hospitals in Tanzania. Study-related laboratory assessments were performed on the day of (CBC and CD4 lymphocyte count) or within 2 weeks of (HIV-1 RNA level) sample receipt and were made immediately available to clinicians to assist with clinical care.

\section{Statistical Analysis}

Statistical analyses were conducted using Stata software, version 10.0 (StataCorp, College Station, TX). The primary outcome was virologic failure, defined as HIV-1 RNA level $\geq 400$ copies per milliliter. Participant characteristics at time of study enrollment were stratified by HIV-1 RNA level $<$ or $\geq 400$ copies per milliliter and compared using Pearson $\chi^{2}$ and Wilcoxon rank-sum tests as appropriate. Bivariable analyses were performed to identify associations with virologic failure for all enrolled children. Both bivariable analyses and multivariate logistic regression were performed on select variables to identify predictors of first-line failure. The number of variables in the multivariate model was limited to 1 per 10 virologic failure events, and variables were preselected by an expert in the field blinded to preliminary results to limit overfitting in the model. ${ }^{24}$ Performance of currently recommended clinical and immunologic criteria in identifying children with virologic failure was assessed using sensitivity, specificity, and positive and negative predictive values. Receiver operating characteristic (ROC) curves were plotted for the currently recommended clinical and immunological criteria and those criteria in combination with significant predictors from the logistic regression model. Areas under ROC curves for the resulting monitoring strategies were compared using STATA 10.0 rocgold. Standard errors for areas under ROC curves were calculated using the Hanley method, and Sidak method was used to adjust for multiple comparisons.

\section{Research Ethics}

The study protocol was approved by an Institutional Review Board of Duke University Medical Center, the Kilimanjaro Christian Medical Centre Research Ethics Committee, and the Tanzania National Institute for Medical Research National Medical Research Coordinating Committee.

\section{Role of the Funding Source}

Study sponsors played no role in study design, data collection or analysis, article writing, or submission for publication.

\section{RESULTS}

\section{Study Population}

Two hundred six children were enrolled between October 2008 and June 2009, representing 84\% of the clinic population eligible for the study (Fig. 1). Of the 38 eligible children who were not enrolled, 8 declined participation and 8 came to clinic without a parent or guardian. In 4 cases, the caretaker came alone to collect medications, and in 2 cases, the parent or guardian did not speak Kiswahili. The remaining 16 children attended clinic sporadically and were never selected on their visits. Enrollment was closed after there was less than 1 eligible child in attendance per week for greater than 1 week of clinic.

Characteristics of the 206 participants at the time of study enrollment are described in Table 2. The median [intraquartile range (IQR)] age was $8.1(5.4-11.2)$ years, and median (IQR) duration of ART before enrollment was 2.4 (1.1-3.3) years. Five children (2.4\%) (ranging from 1.3-2.9 years of age) had received single-dose nevirapine for prevention of perinatal transmission of HIV. At enrollment, 182 participants $(88.3 \%)$ were receiving a non-nucleoside reverse transcriptase inhibitor. Nevirapine-based ART was being taken by 121 children (58.7\%), 71 (34.5\%) in combination with zidovudine and lamivudine and $50(24.3 \%)$ in combination with stavudine and lamivudine. Efavirenz-based ART was being taken by 61 children (29.6\%), $57(27.7 \%)$ in combination with zidovudine and lamivudine and $4(1.9 \%)$ in combination with stavudine and lamivudine. Twenty-four children $(11.6 \%)$ were using protease inhibitor-based ART, and $23(95.8 \%)$ of those 24 were receiving it as second-line therapy. The remaining 183 children were on first-line ART.

\section{Virologic Failure}

Sixty-five children $(31.6 \%)$ demonstrated virologic failure (HIV-1 RNA $\geq 400$ copies $/ \mathrm{mL}$ ) (Fig. 1). Of the participants experiencing failure, $57(87.7 \%)$ were on first-line therapy and the remaining $8(12.3 \%)$ were on second-line therapy. Compared with children with virologic suppression, children with virologic failure were significantly younger [7.7 (4.5-10.1) versus $9.0(6.0-11.5)$ years; $P=0.02]$, and were more likely to be taking a nevirapine-based regimen versus any other regimen [odds ratio $(\mathrm{OR})=1.9 ; P=0.04$ ] and versus an efavirenz-based regimen $(\mathrm{OR}=2.4 ; P=0.02)$ (Table 2). A history of WHO Stage IV disease $(\mathrm{OR}=2.2 ; P=0.01)$ was also associated with virologic failure in this cohort. Among 


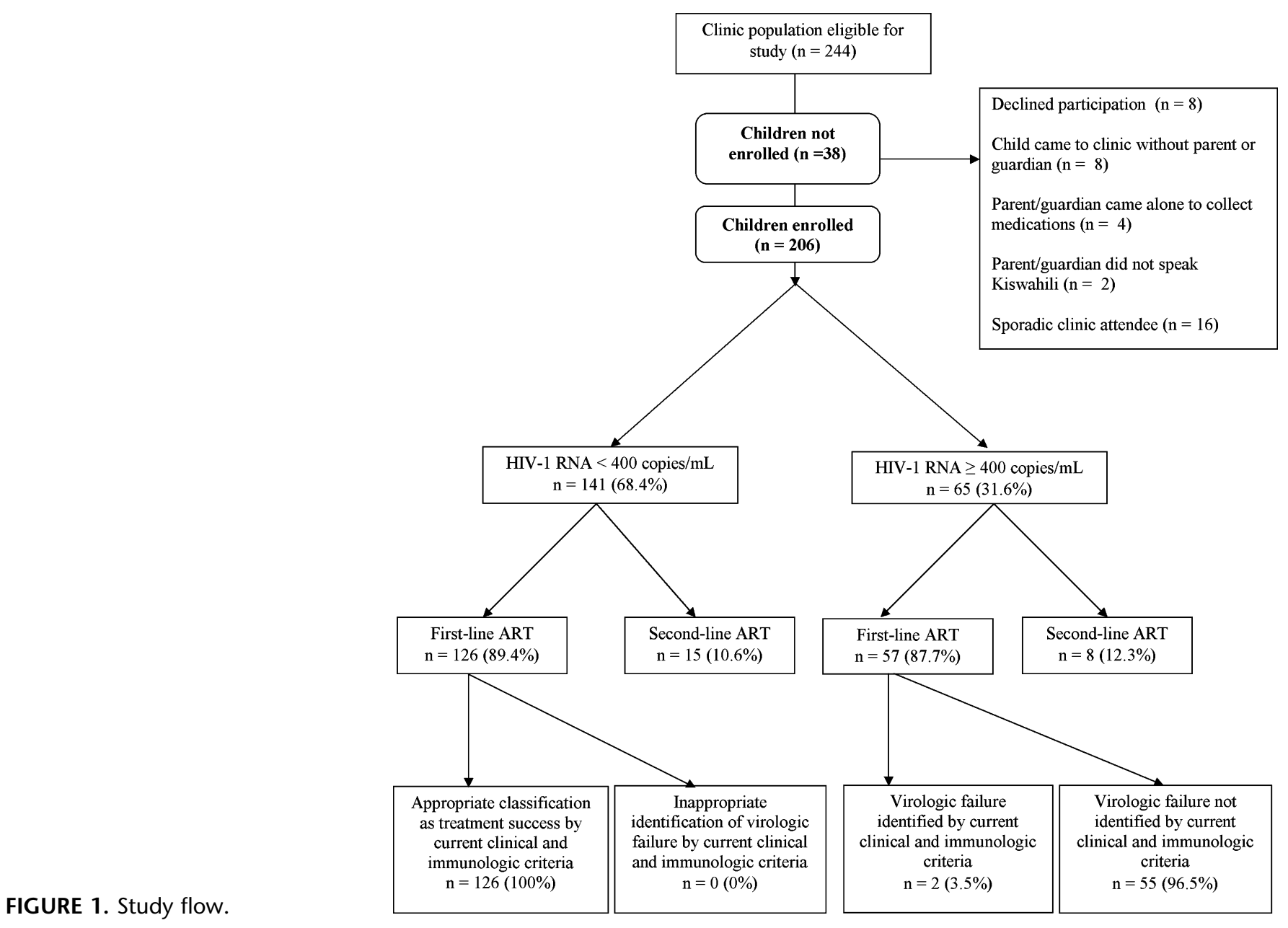

those ever treated for tuberculosis, children with virologic failure were more likely to have taken antituberculosis therapy and ART simultaneously $(\mathrm{OR}=2.4 ; P=0.04)$ as opposed to sequentially.

A greater proportion of children with virologic failure had ever received care at an HIV care center in addition to $\mathrm{KCMC}(\mathrm{OR}=2.1 ; P=0.04)$ and had a history of at least 1 adult on ART in the household ( $\mathrm{OR}=2.0 ; P=0.03$ ). Physician documentation of maladherence $(\mathrm{OR}=3.2 ; P<0.01)$ was found more frequently among participants with virologic failure, whereas family report of missed doses was not significantly associated. Full disclosure of HIV status to children aged 7 and older was protective against virologic failure $(\mathrm{OR}=0.26, P=0.02)$.

Study enrollment $\mathrm{CD} 4 \%<25 \%(\mathrm{OR}=2.9, P<0.01)$ and CD4 lymphocyte count $<200$ cells per milliliter (OR undefined, $P<0.01$ ) were both associated with virologic failure in this cohort. For children with chart-abstracted CD4 lymphocyte measurements available in addition to study values (Table 3), a decline to below pre-ART CD4\% nadir after greater than 6 months of ART was associated with virologic failure (OR undefined, $P=0.04$ ), whereas a decline to below pre-ART nadir CD4 lymphocyte count (assessed among children $\geq 5$ years at ART start) was not. A single decline in
CD4 lymphocyte count by $>30 \%$ between consecutive tests $(\mathrm{OR}=2.1, P=0.05)$ or 2 declines in $\mathrm{CD} 4$ lymphocyte count by $>10 \%$ over consecutive tests $(\mathrm{OR}=3.5, P=<0.01)$ were significantly associated with virologic failure among the 159 children aged $\geq 5$ years at study enrollment. A decline in $\mathrm{CD} 4 \%$ from post-treatment peak to study $\mathrm{CD} 4 \%$ was not significantly associated with virologic failure.

In evaluating virologic failure on first-line therapy (Table 4), bivariable analysis of endpoints independently selected before data analysis revealed enrollment CD4\% $<$ $25 \%(\mathrm{OR}=3.2 ; P<0.01)$ to be significantly predictive of failure and physician documentation of maladherence $(\mathrm{OR}=$ 2.3; $P=0.07)$ trended toward significance. On multivariate analysis, both $\mathrm{CD} 4 \%<25 \%(\mathrm{OR}=3.7 ; P<0.01)$ and physician documentation of maladherence $(\mathrm{OR}=5.0 ; P=$ 0.01 ) were significantly predictive of virologic failure. Family reported adherence, a decline from post-treatment peak $\mathrm{CD} 4 \%$, and recent change in weight-for-age $Z$ score were not predictive of failure in this group.

Analyses with virologic failure defined as $\geq 1000$ copies per milliliter [found in $60(29 \%)$ participants] revealed few differences across all assessments. Care at another center in addition to KCMC was no longer significant. Physician report of maladherence was significant in both bivariable $(\mathrm{OR}=2.4$, 
TABLE 2. Demographic and Clinical Characteristics at Enrollment of Children Receiving ART at the Kilimanjaro Christian Medical Centre in Moshi, Tanzania, October 2008-June 2009. Participants Stratified by Virologic Failure (HIV-1 RNA $\geq 400$ Copies/mL)

\begin{tabular}{|c|c|c|c|c|}
\hline Characteristic & All patients $n=206$ & HIV-1 RNA $<400 n=141$ & HIV-1 RNA $\geq 400 n=65$ & $P *$ \\
\hline Male sex & $98(47.6)$ & $68(48.2)$ & $30(46.2)$ & 0.78 \\
\hline Age in years, median (IQR) & $8.7(5.4-11.2)$ & $9.0(6.0-11.5)$ & $7.7(4.5-10.1)$ & 0.02 \\
\hline Transferred or care in $>1$ center $\dagger$ & $37(18.0)$ & $20(14.2)$ & $17(26.2)$ & 0.04 \\
\hline Duration of ART in years, median (IQR) & $2.4(1.1-3.3)$ & $2.4(1.2-3.3)$ & $2.1(1.1-3.4)$ & 0.96 \\
\hline \multicolumn{5}{|l|}{ Current ART Regimen } \\
\hline Nevirapine-based & $121(58.7)$ & $76(53.9)$ & $45(69.2)$ & 0.04 \\
\hline Efavirenz-based & $61(29.6)$ & $49(34.8)$ & $12(18.5)$ & 0.02 \\
\hline Protease inhibitor-based & $24(11.6)$ & $16(11.4)$ & $8(12.3)$ & 0.82 \\
\hline \multicolumn{5}{|l|}{ Prior medication change $\neq$} \\
\hline None & $158(76.7)$ & $109(77.3)$ & $49(75.4)$ & 0.60 \\
\hline 1 change & $44(21.4)$ & $29(20.7)$ & $15(23.1)$ & 0.72 \\
\hline 2 changes & $10(4.8)$ & $8(5.7)$ & $2(3.1)$ & 0.51 \\
\hline Perinatal nevirapine exposure & $5(2.4)$ & $1(0.7)$ & $4(6.2)$ & 0.04 \\
\hline Caregiver change in past year & $24(12.2)$ & $18(13.3)$ & $6(9.8)$ & 0.64 \\
\hline Mother deceased & $89(43.2)$ & $66(46.8)$ & $23(35.4)$ & 0.17 \\
\hline Father deceased & $69(33.5)$ & $50(35.5)$ & $19(29.2)$ & 0.43 \\
\hline Primary caregiver not biologic parent & $94(45.6)$ & $67(47.5)$ & $26(40.0)$ & 0.37 \\
\hline No. of children $<18$ years in household, median (IQR) & $2(1-3)$ & $2(1-3)$ & $2(1-3)$ & 0.73 \\
\hline Time to clinic in hours, median (IQR) & $1.5(0.8-2.0)$ & $1.5(0.8-2.0)$ & $1.5(1.0-2.5)$ & 0.98 \\
\hline Cost of travel to clinic in USD, median (IQR) $\S$ & $1.8(0.9-3.8)$ & $1.9(0.9-3.8)$ & $1.8(0.9-3.1)$ & 0.60 \\
\hline \multicolumn{5}{|l|}{ Disclosure of HIV status } \\
\hline Child $\geq 7$ years aware $\|$ & $48(36.9)^{\|}$ & $40(72.3)^{\|}$ & $8(16.7)^{\|}$ & 0.02 \\
\hline All others in household $\geq 13$ years aware & $110(53.7)$ & $73(51.8)$ & $37(57.8)$ & 0.45 \\
\hline \multicolumn{5}{|l|}{ Seropositivity in household } \\
\hline HIV-infected but no ART & $21(10.2)$ & $16(11.4)$ & $5(7.7)$ & 0.62 \\
\hline At least one adult on ART & $88(42.7)$ & $53(37.6)$ & $35(53.8)$ & 0.03 \\
\hline No one else with HIV in Household & $87(42.2)$ & $67(47.5)$ & $20(30.8)$ & 0.02 \\
\hline \multicolumn{5}{|l|}{ Maladherence } \\
\hline Family report & $46(22.3)$ & $29(20.6)$ & $17(26.2)$ & 0.45 \\
\hline Physician documentation & $31(15.3)$ & $14(10.1)$ & $17(26.6)$ & $<\mathbf{0 . 0 1}$ \\
\hline \multicolumn{5}{|l|}{ Tuberculosis status } \\
\hline Ever treated & $108(52.4)$ & $73(51.8)$ & $35(53.8)$ & 0.52 \\
\hline TB and ART taken Simultaneously & $59(54.1)$ & $35(47.3)$ & $24(68.6)$ & 0.04 \\
\hline \multicolumn{5}{|l|}{ WHO Stage } \\
\hline Stage II & $29(14.1)$ & $22(15.6)$ & $7(10.8)$ & 0.40 \\
\hline Stage III & $118(57.3)$ & $86(61.0)$ & $32(49.2)$ & 0.13 \\
\hline Stage IV & $56(27.2)$ & $31(22.0)$ & $25(38.5)$ & 0.01 \\
\hline Weight-for-age Z-score, median (IQR) & $-1.4(-2.2--0.5)$ & $-1.4(-2.3--0.5)$ & $-1.2(-2.2--0.3)$ & 0.37 \\
\hline Pre-ART CD4\%, median (IQR) & $12(6-14)$ & $12(6-14)$ & $11(6-11)$ & 0.90 \\
\hline \multicolumn{5}{|l|}{ Enrollment laboratory values } \\
\hline CD4\%, median (IQR) & $28(21-34)$ & $30(24-35)$ & $25(16-28)$ & $<0.01$ \\
\hline $\mathrm{CD} 4 \%<25 \%$ & $66(33.2)$ & $35(25.6)$ & $31(50.0)$ & $<0.01$ \\
\hline CD4 count, median (IQR) & $834(543-1154)$ & $926(658-1162)$ & $615(437-1151)$ & $<\mathbf{0 . 0 1}$ \\
\hline CD4 count $<200$ cells $/ \mathrm{mL}$ & $4(2.0)$ & $0(0.0)$ & $4(6.3)$ & $<0.01$ \\
\hline Hemoglobin, median (IQR) & $12.0(11.1-12.8)$ & $12.1(11.4-12.8)$ & $11.8(10.9-12.6)$ & 0.11 \\
\hline MCV, median (IQR) & $94(86-102)$ & $95(86-102)$ & $92(83-101)$ & 0.16 \\
\hline Platelet count, median (IQR) & $346(289-411)$ & $347(292-417)$ & $332(268-400)$ & 0.12 \\
\hline \multicolumn{5}{|c|}{$\begin{array}{l}\text { Data are number (\%) of patients unless otherwise indicated. IQR, interquartile range; ART, antiretroviral therapy; USD, United States dollars; SD, standard deviation; MCV, mean } \\
\text { puscular volume. }\end{array}$} \\
\hline
\end{tabular}


TABLE 3. Immunologic Associations With Virologic Failure after Initiation of ART

\begin{tabular}{|c|c|c|c|c|}
\hline All participants & $\begin{array}{c}\mathrm{N} \text { with finding/N } \\
\text { with available data }(\%)\end{array}$ & HIV-1 RNA $<400$ & HIV-1 RNA $\geq 400$ & $P *$ \\
\hline Study CD $4 \%$ less than post-treatment peak & $119 / 203(58.6)$ & $79 / 140(56.4)$ & $40 / 63(63.5)$ & 0.63 \\
\hline Any $\mathrm{CD} 4 \%$ less than pre-treatment nadir $\dagger$ & $2 / 44(4.6)$ & $0 / 29(0.0)$ & 2/15 (13.3) & 0.04 \\
\hline \multicolumn{5}{|l|}{ Participants $\geq 5$ years old at ART start } \\
\hline Any CD4 count less than pre-treatment nadir $\dagger$ & $19 / 140(13.6)$ & $12 / 102(11.6)$ & $7 / 38(18.4)$ & 0.40 \\
\hline Decline in CD 4 count by $>30 \%$ over two consecutive tests & $46 / 157(29.3)$ & $29 / 113(24.8)$ & $18 / 44(40.9)$ & 0.05 \\
\hline Two declines in CD4 count $>10 \%$ over three consecutive tests & $21 / 136(15.4)$ & $10 / 99(10.1)$ & $11 / 37(29.7)$ & $<\mathbf{0 . 0 1}$ \\
\hline
\end{tabular}

*Significance tests for comparisons between HIV-1 RNA $<$ or $\geq 400$ copies/ml based on Pearson's chi-square.

$\dagger$ After 6 months of ART.

\$Only one child over age 5 had a consective decline in both CD4 count and percent; this child had HIV-1 RNA $>400 \mathrm{copies} / \mathrm{mL}$.

$P=0.04)$ and multivariate analyses $(\mathrm{OR}=4.1, P=0.01)$ of virologic failure on first-line therapy.

\section{Strategies for Identifying Virologic Failure}

Currently recommended clinical and immunologic criteria demonstrated $3.5 \%$ sensitivity in this cohort, correctly classifying 2 of 57 subjects (3.5\%) with virologic failure on first-line ART (Table 5). All 126 participants with plasma HIV1 RNA $<400$ copies per milliliter were correctly identified by these criteria as not requiring regimen change.

By incorporating physician documentation of maladherence and $\mathrm{CD} 4 \%<25 \%$ into current monitoring strategies, sensitivity in detecting failure increased to $57.1 \%$, with a decrease in specificity to $69.6 \%$. Thirty-two of 57 children $(56.1 \%)$ with first-line failure were correctly identified with this method. However, 38 of 126 children (30.2\%) with virologic suppression were incorrectly classified as experiencing virologic failure. The area under the ROC curve for currently recommended clinical and immunologic criteria was 0.52 [standard error $(\mathrm{SE})=0.05$ ]. Including $\mathrm{CD} 4 \%<25 \%$ increased the area under ROC curve to $0.64(\mathrm{SE}=0.05)$ and including both predictors produced an area of $0.63(\mathrm{SE}=0.05)$.

\section{DISCUSSION}

As antiretroviral access continues to expand in resourcelimited settings, monitoring response to therapy is becoming an increasingly critical issue. Virologic and immunologic testing are central to guidelines for monitoring ART in resource-replete settings, and plasma HIV-1 RNA testing has been shown to be cost-effective and improve patient outcomes in resource-rich countries. ${ }^{25}$ With only 1 second-line regimen available in many resource-limited areas, timely detection of virologic failure is likely to be crucial in preventing resistance mutations that could jeopardize the long-term effectiveness of currently available ART. ${ }^{17-19}$ Clinical and immunological monitoring of children in resource-limited settings may be even more challenging than in adults, given children's baseline increased risk of infections, the normal age-related decline in CD4 lymphocyte counts, and frequent lack of availability of $\mathrm{CD} 4 \%$. Yet in our cohort and in others in sub-Saharan Africa, children seem to be at high risk for virologic failure, with published prevalence ranging from $19.7 \%$ in KwaZulu-Natal, South Africa, to $50 \%$ in Cote d'Ivoire. ${ }^{5,26}$ Therefore, close monitoring of response to ART may be particularly important in children, especially given the goal of lifelong therapy from infancy through adulthood.

Several clinical and immunologic features correlated with virologic failure in our cohort have been identified in other studies, including CD4\% $<25 \%,{ }^{4}$ younger age at ART initiation, ${ }^{3,27}$ and use of nevirapine vs. efavirenz-based ART. $^{2,28}$ The superiority of efavirenz-based regimens has been suggested by cohort studies in adults, ${ }^{29,30}$ but clinical trial data are not available for children. Furthermore, efavirenz is not currently recommended for children less than 3 years of age, a group that seems to be at higher risk for virologic

TABLE 4. Predictors of Virologic Failure (HIV-1 RNA $\geq 400$ Copies $/ \mathrm{mL}$ ) In a Pediatric Tanzanian Cohort After at Least Six Months of First-line ART $(n=183)$

\begin{tabular}{|c|c|c|c|c|}
\hline \multirow[b]{2}{*}{ Variable } & \multicolumn{2}{|c|}{ Bivariate analysis } & \multicolumn{2}{|c|}{ Multivariate analysis* } \\
\hline & Unadjusted OR (95\% CI) & $P$ & Adjusted OR (95\% CI) & $P$ \\
\hline \multicolumn{5}{|l|}{ Maladherence } \\
\hline Family report & $1.0(0.48-2.2)$ & 0.95 & $0.6(0.21-1.8)$ & 0.37 \\
\hline Physician documentation & $2.3(0.95-5.6)$ & 0.07 & $5.0(1.4-17.3)$ & 0.01 \\
\hline Drop in CD4\% from peak & $1.4(0.71-2.6)$ & 0.34 & $1.7(0.76-3.9)$ & 0.19 \\
\hline Enrollment CD4\% $<25 \%$ & $3.2(1.7-6.3)$ & $<0.01$ & $3.7(1.7-8.4)$ & $<0.01$ \\
\hline Weight-for-age growth velocity $\dagger$ & $1.1(0.61-1.9)$ & 0.78 & $1.0(0.56-1.9)$ & 0.92 \\
\hline Hemoglobin at enrollment & $0.98(0.95-1.0)$ & 0.04 & $0.98(0.96-1.0)$ & 0.51 \\
\hline
\end{tabular}


TABLE 5. Performance Characteristics of Strategies for Predicting Virologic Failure (HIV-1 RNA $\geq 400$ Copies/mL) in a Pediatric Tanzanian Cohort on First-line ART

\begin{tabular}{|c|c|c|c|c|c|}
\hline Strategy & Area under ROC (95\% CI) & Sensitivity (95\% CI) & Specificity (95\% CI) & PPV (95\% CI) & NPV (95\% CI) \\
\hline $\begin{array}{l}\text { Currently recommended clinical } \\
\text { and immunologic criteria }\end{array}$ & $0.52(0.49-0.54)$ & $3.5(0.4-12.1)$ & $100(97.1-100)$ & $100(15.8-100)$ & $69.6(62.4-76.2)$ \\
\hline $\begin{array}{l}\text { Current criteria plus physician } \\
\text { documentation of maladherence }\end{array}$ & $0.57(0.51-0.63)$ & $23.2(13.0-36.4)$ & $90.4(83.8-94.9)$ & $52.0(31.3-72.2)$ & $72.4(64.7-79.3)$ \\
\hline Current criteria plus CD $4 \%<25 \%$ & $0.64(0.56-0.71)$ & $50.9(37.3-64.4)$ & $77.0(68.6-84.0)$ & $50.0(36.6-63.4)$ & $77.6(69.3-84.6)$ \\
\hline $\begin{array}{l}\text { Current criteria, documented } \\
\text { maladherence, and CD } 4 \%<25 \%\end{array}$ & $0.63(0.56-0.71)$ & $57.1(43.2-70.3)$ & $69.6(60.7-77.5)$ & $45.7(33.7-58.1)$ & $78.4(69.6-85.6)$ \\
\hline
\end{tabular}

ROC, receiver operating characteristic; CI, confidence interval.

failure. Use of nevirapine-based regimens in children aged less than 3 years may have contributed to our finding that a history of receipt of ART in conjunction with antituberculosis therapy was associated with virologic failure. This merits further exploration in additional studies.

We found a significant association between virologic failure and physician documentation of maladherence but not with family-reported maladherence. The lack of correlation between family report and virologic outcomes is consistent with prior studies., ${ }^{2,2}$ In our setting, the finding underscores the importance of the use of the national HIV care flow sheet at each visit and optimized attention to adherence and vigilance for treatment failure among families with any physician documentation of missed doses.

Full disclosure of HIV status by caregivers to their school-aged children (in this case, we defined school-age as 7 years and older) was associated with a protective effect against virologic failure. These data suggest that techniques to improve rates of caregiver disclosure might improve virologic outcomes in children. The finding that having another adult on ART in the household was associated with virologic failure was surprising. Poorer health or economic status among caretakers receiving ART compared with non-HIV-infected caretakers or those who were at early stages of HIV infection may have been responsible for this association but were not assessed in our study.

Currently recommended clinical and immunologic criteria demonstrated poor performance in our cohort, identifying very few participants with virologic failure. Their excellent specificity avoided unnecessary treatment changes for children who were virologically suppressed, but low sensitivity left $96.5 \%$ of patients with virologic failure on failing first-line regimens. The addition of 2 factors highly correlated with virologic failure in our multivariate analysis markedly reduced the specificity of the model and, if used clinically, would risk over ascertainment of virologic failure. Although we analyzed only a limited number of prespecified factors in our multivariate model, use of other factors that were significantly associated with virologic failure on bivariable analysis would be unlikely to improve the sensitivity of clinical and immunologic detection to a clinically meaningful level. For example, rapid or repeated declines in CD4 count among children aged 5 or older were associated with but were not found exclusively among children with virologic failure.
There are several limitations to this study. The crosssectional nature of the study design did not allow for multiple plasma HIV-1 RNA measurements, and blips in HIV-1 RNA level are known to occur. ${ }^{31}$ However, analysis of our data points with an outcome of $\geq 1000$ copies per milliliter did not substantially change findings. The study design also did not allow us to assess whether the sensitivity of current guidelines changes with the length of time a child experiences unsuppressed viremia nor could we assess the degree to which longterm clinical outcomes and response to second-line therapy are impacted by use of clinical and immunologic versus virologic monitoring strategies. In addition, the low number of children meeting clinical guidelines for failure at study enrollment may have been influenced by death, loss to follow-up of clinic attendees, and use of second-line therapy. Further, retrospective laboratory measurements abstracted from charts were performed in a variety of clinical laboratories, some of which do not adhere to the same rigorous quality control standards as the KCMC Biotechnology Laboratory where all enrollment laboratories were performed. Although this lack of consistency in laboratory capacity does represent a limitation, it is more reflective of real-world circumstances under which most children in resource-limited settings receive HIV-related care. Finally, because the WHO guidelines were written specifically to address first-line failure, our multivariate model included only children on first-line therapy.

Despite these limitations, this study also has important strengths. Our cohort has taken ART for a mean of 2.4 years, which is substantially longer than many current studies examining virologic outcomes in children. Detailed sociodemographic and clinical information was collected on the cohort, and differences between subjects on failing and suppressive regimens may direct future studies examining underlying reasons for virologic failure. Furthermore, as far as we are aware, this study is the first to examine the effectiveness of currently recommended clinical and immunologic criteria in monitoring children for virologic failure on ART.

\section{CONCLUSIONS}

This study has demonstrated poor performance of currently recommended clinical and immunologic criteria in detecting virologic failure among children in a resource-limited setting. Resistance mutations and subsequent second-line 
failure shown to develop in patients remaining on failing regimens represent a barrier to long-term effectiveness of ART, which makes evaluation and improvement of the current clinical and immunologic monitoring strategy a critical issue. ${ }^{18}$ Ultimately, there is an urgent need for affordable techniques for measuring plasma HIV-1 RNA level that could be applied in resource-limited settings.

\section{ACKNOWLEDGMENTS}

Special thanks to the clinic staff of the Child Centered Family Care Clinic, especially Hedwiga Mrema, Elissiana Ndossa, George Chambo, and Dorice Kiama for their hard work and dedication to the project.

\section{REFERENCES}

1. UNAIDS. Epidemiological fact sheet on HIV and AIDS: United Republic of Tanzania. Available at: http://www.unaids.org/en/KnowledgeCentre/ HIVData/Epidemiology/epifactsheets.asp. Accessed July 6, 2009.

2. Puthanakit T, Oberdorfer A, Akarathum N, et al. Efficacy of highly active antiretroviral therapy in HIV-infected children participating in Thailand's National Access to Antiretroviral Program. Clin Infect Dis. 2005;41: 100-107.

3. Jaspan HB, Berrisford AE, Boulle AM. Two-year outcomes of children on non-nucleoside reverse transcriptase inhibitor and protease inhibitor regimens in a South African pediatric antiretroviral program. Pediatr Infect Dis J. 2008;27:993-998.

4. Resino S, MBellon J, Gurbindo D, et al. Viral load and CD4+ T lymphocyte response to highly active antiretroviral therapy in human immunodeficiency virus type 1-infected children: an observational study. Clin Infect Dis. 2003;37:1216-1225.

5. Fassinou P, Elenga N, Rouet F, et al. Highly active antiretroviral therapies among HIV-1-infected children in Abidjan, Cote d'Ivoire. AIDS. 2004;18: 1905-1913.

6. Sutcliffe CG, van Dijk JH, Bolton C, et al. Effectiveness of antiretroviral therapy among HIV-infected children in sub-Saharan Africa. Lancet Infect Dis. 2008;8:477-489.

7. Petti CA, Polage CR, Quinn TC, et al. Laboratory medicine in Africa: a barrier to effective health care. Clin Infect Dis. 2006;42:377-382.

8. World Health Organization (WHO). Guidelines for the use of antiretroviral agents in pediatric HIV infection. Available at: http://aidsinfo.nih. gov/ContentFiles/PediatricGuidelines.pdf. Accessed July 13, 2009.

9. World Health Organization (WHO). Antiretroviral therapy for HIV infection in infants and children: towards universal access. Available at: http://www.who.int/hiv/pub/guidelines/art/en/. Accessed July 13, 2009.

10. World Health Organization (WHO). Priority interventions: HIV/AIDS prevention, treatment, and care in the health sector. Available at: http:// www.who.int/hiv/pub/priorityinterventions/en/index.html. Accessed July 13, 2009.

11. van Oosterhout JJ, Brown L, Weigel R, et al. Diagnosis of antiretroviral therapy failure in Malawi: poor performance of clinical and immunological WHO criteria. Trop Med Int Health. 2009;14:856-861.

12. Kantor R, Diero L, Delong A, et al. Misclassification of first-line antiretroviral treatment failure based on immunological monitoring of HIV infection in resource-limited settings. Clin Infect Dis. 2009;49:454-462.

13. Reynolds SJ, Nakigozi G, Newell K, et al. Failure of immunologic criteria to appropriately identify antiretroviral treatment failure in Uganda. AIDS. 2009;23:697-700

14. Mee P, Fielding KL, Charalambous S, et al. Evaluation of the WHO criteria for antiretroviral treatment failure among adults in South Africa. AIDS. 2008;22:1971-1977.
15. Phillips AN, Pillay D, Miners AH, et al. Outcomes from monitoring of patients on antiretroviral therapy in resource-limited settings with viral load, CD4 cell count, or clinical observation alone: a computer simulation model. Lancet. 2008;371:1443-1451.

16. Coutinho A, Mermin J, Ekwaru J, et al. Utility of routine viral load, CD4 cell count, and clinical monitoring among HIV-infected adults in Uganda: a randomized trial. Presented at: 15th Conference on Retroviruses and Opportunistic Infections; February 3-6, 2008; Boston, MA.

17. Petersen ML, van der Laan MJ, Napravnik S, et al. Long-term consequences of the delay between virologic failure of highly active antiretroviral therapy and regimen modification. AIDS. 2008;22: 2097-2106.

18. Hosseinipour MC, van Oosterhout JJ, Weigel R, et al. The public health approach to identify antiretroviral therapy failure: high-level nucleoside reverse transcriptase inhibitor resistance among Malawians failing firstline antiretroviral therapy. AIDS. 2009;23:1127-1134.

19. Gupta R, Hill A, Sawyer W, et al. Drug resistance after virological failure of first-line HAART in resource rich and poor settings - a meta-analysis. Presented at: 15th Conference on Retroviruses and Opportunistic Infections; February 3-6, 2004; Boston, MA.

20. National AIDS Control Programme. National guidelines for the management of HIV and AIDS, third edition. Available at: http://www.nacp.go.tz/ modules/doc_sm/docs_by_type.php?tbltype_doctype=Guidelines. Accessed July $29,2009$.

21. World Health Organization (WHO). WHO case definitions of HIV for surveillance and revised clinical staging and immunologic classification of HIV-related disease in adults and children. Available at: http:// www.who.int/hiv/pub/vct/hivstaging/en/index.html. Accessed July 13, 2009.

22. World Health Organization (WHO). Report of the WHO Technical Reference Group. Presented at: Paediatric HIV/ART Care Guideline Group Meeting; 2008; Geneva, Switzerland. Available at: http://www. who.int/hiv/pub/paediatric/WHO_Paediatric_ART_guideline_rev_mreport_ 2008.pdf. Accessed 1 October, 2009.

23. Crump JA, Scott LE, Msuya E, et al. Evaluation of the Abbott m2000rt RealTime HIV-1 assay with manual sample preparation compared with the ROCHE COBAS AmpliPrep/AMPLICOR HIV-1 MONITOR v1.5 using specimens from East Africa. J Virol Methods. 2009;162:218-222.

24. Babyak MA. What you see may not be what you get: a brief, nontechnical introduction to overfitting in regression-type models. Psychosom Med. 2004;66:411-421.

25. Hornberger J, Holodniy M, Robertus K, et al. A systematic review of costutility analyses in HIV/AIDS: implications for public policy. Med Decis Making. 2007;27:789-821.

26. Reddi A, Leeper SC, Grobler AC, et al. Preliminary outcomes of a paediatric highly active antiretroviral therapy cohort from KwaZuluNatal, South Africa. BMC Pediatr. 2007;7:13.

27. Kekitiinwa A, Lee KJ, Walker AS, et al. Differences in factors associated with initial growth, CD4, and viral load responses to ART in HIV-infected children in Kampala, Uganda, and the United Kingdom/Ireland. $J$ Acquir Immune Defic Syndr. 2008;49:384-392.

28. Kamya MR, Mayanja-Kizza H, Kambugu A, et al. Predictors of long-term viral failure among ugandan children and adults treated with antiretroviral therapy. J Acquir Immune Defic Syndr. 2007;46:187-193.

29. van Leth F, Phanuphak P, Ruxrungtham K, et al. Comparison of first-line antiretroviral therapy with regimens including nevirapine, efavirenz, or both drugs, plus stavudine and lamivudine: a randomised open-label trial, the 2NN Study. Lancet. 2004;363:1253-1263.

30. Starr SE, Fletcher CV, Spector SA, et al. Combination therapy with efavirenz, nelfinavir, and nucleoside reverse-transcriptase inhibitors in children infected with human immunodeficiency virus type 1. Pediatric AIDS Clinical Trials Group 382 Team. N Engl J Med. 1999;341:1874-1881.

31. Nettles RE, Kieffer TL, Kwon P, et al. Intermittent HIV-1 viremia (Blips) and drug resistance in patients receiving HAART. JAMA. 2005;293: $817-829$. 